\title{
Inflammatory Pseudotumor of Spleen: A Case Report
}

\author{
Omar Toumi ${ }^{1}$, Houssem Ammar ${ }^{1 *}$, Amine Chhaidar ${ }^{2}$, Rahul Gupta ${ }^{3}$, Ibtissem Korbi ${ }^{1}$, Mohamed \\ Nasr $^{1}$, Faouzi Noomen ${ }^{1}$ and Khadija Zouari ${ }^{1}$ \\ ${ }^{1}$ Department of Surgery, Hospital fattouma bourguiba,Monastir, Tunisia \\ ${ }^{2}$ Department of surgery, Hospital Sahloul, Sousse, Tunisia \\ ${ }^{3}$ Department of Gastrointestinal Surgery, Synergy Institute of Medical Sciences, Dehradun, India
}

"Corresponding Author: Houssem Ammar, Department of surgery, Fattouma Bourguiba Hospital, Monastir, Tunisia, Tel: +21652688551; E-mail: hosshoss24@hotmail.fr

Received: 19 July 2017; Accepted: 25 July 2017; Published: 31 July 2017

\begin{abstract}
Introduction: Inflammatory pseudotumor (IPT) is a rare benign disease which presents as tumor like mass mimicking malignancy. The most common sites are lungs, liver, orbits and gastrointestinal tract. Splenic IPT is very rare.

Presentation of Case: A 41-year-old woman presented with the complaints of left upper quadrant pain radiating to the back for a year. Abdominal ultrasound and computed tomography (CT) revealed a hypodense lesion of approximately $5 \mathrm{~cm}$ located in the splenic parenchyma near the hilum. Hydatid serology was negative. The patient was treated with splenectomy due to diagnostic dilemma and final diagnosis of IPT was made on histopathological examination of the resected spleen.

Discussion: Splenic IPT usually affects middle aged women presenting as incidental finding on abdominal imaging. It is most often isolated but at times may be associated with IPT at other sites probably suggesting a systemic etiology. Radiological findings are non-specific leading to diagnostic dilemma. Splenectomy is useful for treating symptomatic cases and helps in making the accurate diagnosis in cases of diagnostic dilemma.

Conclusion: Splenic IPT is a rare, benign condition which should be included in the differential diagnosis while evaluating a splenic mass.
\end{abstract}

Keywords: Pseudotumor; Spleen; Splenectomy; Case report 
Abbreviations: CT: computed tomography; EBV: Epstein Barr virus; IPT: inflammatory pseudotumor; MRI: Magnetic resonance imaging

\section{Introduction}

Inflammatory pseudotumors (IPT) are mass-like lesions of unknown etiology characterized by features of nonspecific inflammation and mesenchymal proliferation on histology [1]. They can occur anywhere in the body with the most common sites being lung, liver, gastrointestinal tract and orbits [2]. The clinical significance of this rare condition lies in the fact that they mimic malignant tumor on preoperative imaging. Because of the diagnostic dilemma, most of the patients undergo radical surgery with IPT being diagnosed on the histological examination. Splenic IPT is an extremely rare condition. Most of the patients are asymptomatic with detection of splenic mass on radiological imaging [1]. With malignant splenic tumor being much more common than benign lesions, most of the patients undergo splenectomy [2]. We report a case of IPT of spleen diagnosed postoperatively on histopathological examination of resected spleen in a 41-year old lady suspected to have splenic tumor on abdominal imaging. This case has been reported in line with the SCARE criteria [3].

\section{Case Presentation}

A 41-year-old woman presented to the outpatient department of our University hospital with complaints of left upper quadrant pain radiating to the back for a year. There were no other associated symptoms. She smoked 10 cigarettes per day for the past 20 years. She had no significant past medical or surgical history. Clinical examination was unremarkable. Routine laboratory tests were normal. Abdominal ultrasound and computed tomography (CT) revealed a hypodense lesion of approximately $5 \mathrm{~cm}$ located in the splenic parenchyma near the hilum. On magnetic resonance imaging (MRI) of the abdomen, the splenic mass was $5.5 \mathrm{~cm}$ in diameter with well-defined boundaries, iso-intense on T1-weighted images and heterogenous on T2-weighted images. On dynamic images the mass showed early but slow enhancement compared to adjoining splenic parenchyma. Hydatid serology was negative.

In view of the above findings, a working diagnosis of malignant splenic tumor was made and open splenectomy was planned. Informed written consent was taken from the patient. Intraoperatively, an encapsulated tumor occupying the central zone of the spleen near the hilum was found. The adjacent organs were not involved. Open splenectomy was performed. The postoperative course was uneventful. The resected spleen was $8 \times 7 \times 4.5 \mathrm{~cm}$ in size weighing 130 grams. On cut section, the lesion appeared as a solid mass without any capsule compressing the adjoining splenic parenchyma. There were some areas of necrosis, hemorrhage at places.

The histological examination showed a multi nodular mass comprising of lymphocytes, plasma cells, eosinophils and histiocytes (Figure 1). No malignant cells were seen. The final diagnosis of an IPT was made. The patient had resolution of the symptoms after the surgery. 


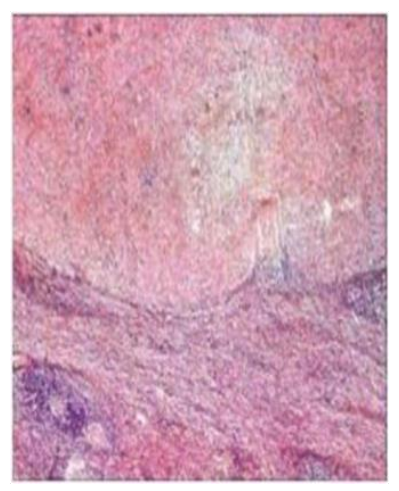

Figure 1: Microscopic examination revealed that the mass consisted of lymphocytes, plasma cells, eosinophils and histiocytes. No tumor cells were seen.

\section{Discussion}

Splenic IPT is usually detected incidentally on radiological imaging performed for other reasons $[4,5]$. It more commonly affects middle and advanced aged adults, especially women [2]. It is a benign condition masquerading as malignant tumor on preoperative imaging. It has been reported to be associated with Epstein Barr Virus (EBV) infection [6], polyclonal hypergamma-globulinemia [7], splenic trauma [8] and tuberculosis [9] but whether they lead to the formation of IPT is not known. In most of the cases, splenic IPT is isolated but at times it may be associated with IPT at other sites probably suggesting a systemic etiology [10]. Clinical signs and symptoms if present are usually nonspecific. Blood investigations are often normal as seen in the present case but there may be some abnormalities like thrombocytopenia, anemia, leukocytosis, hypergamma-globulinemia, elevated C-reactive protein, etc [10].

On ultrasound, splenic IPT appears as a well-defined hypoechoic lesion within the spleen as seen in our case [10]. The lesion is isodense to hypodense on plain CT and hypodense with mild enhancement on contrast enhanced CT similar to the present case [4]. MRI shows the mass as a hypointense heterogenous lesion on T1-weighted images and as a hyperintense lesion with central nodular hypointense areas on T2 and diffusion weighted images [7,11]. But these findings may be present in other splenic masses leading to diagnostic dilemma. Splenectomy helps in making the accurate diagnosis in a timely manner as well as cures the disease in symptomatic cases. However, in patients with high suspicion of benign condition like IPT or very high risk for surgery, percutaneous biopsy can be considered as an alternative to splenectomy $[4,10]$.

\section{Conclusion}

Splenic IPT is a rare, benign condition which should be included in the differential diagnosis while evaluating splenic mass. Definitive diagnosis of IPT can be made only on histopathological examination of biopsy or splenectomy specimen.

\section{Conflict of Interest}

The authors declare that they have no conflict of interest.

\section{Informed Consent}

Written informed consent for the publication of this case report and all accompanying images was obtained from the patient and the patient's family. Copies of the written consents are available for review by the Editor-in-Chief of this journal. 


\section{Funding}

This study was not funded by any organization or institution.

\section{Conflict of Interest}

The authors have no conflict of interest to declare.

\section{Patient Consent}

Informed consent was taken from the patient for case publication.

\section{References}

1. Hassan M, Pujani M, Jairajpuri ZS, et al. Inflammatory Pseudotumor of the Spleen Masquerading as Splenic Malignancy. Oman Med J 31 (2016): 154-157.

2. Ma ZH, Tian XF, Ma J, et al. Inflammatory pseudotumor of the spleen: A case report and review of published cases. Oncol Lett 5 (2013): 1955-1957.

3. Agha RA, Fowler AJ, Saeta A, et al. The SCARE statement: Consensus-based surgical case report guidelines. Int J Surg 34 (2016): 180-186.

4. Yarmohammadi H, Nakamoto D, Faulhaber PF, et al. Inflammatory pseudotumor of the spleen: review of clinical presentation and diagnostic methods. J Radiol Case Rep 5 (2011): 16-22.

5. Hsu CW, Lin CH, Yang TL, et al. Splenic inflammatory pseudotumor mimicking angiosarcoma. World J Gastroenterol 14 (2008): 6421-6424.

6. Zhang M, Lennerz JK, Dehner LP, et al. Granulomatous inflammatory Pseudotumor of the spleen associated with Epstein-Barr Virus. Appl Immunohistochem Mol Morphol 17 (2009): 259-263.

7. Georgia M, Rady K, Prince HM. Inflammatory pseudotumor of the spleen. Hematol Rep 7 (2015): 5905.

8. Ugalde P, Garcia Bernardo C, Granero P, et al. Inflammatory pseudotumor of spleen: a case report. Int J Surg Case Rep 7 (2015): 145-148.

9. Prieto-Nieto MI, Pérez-Robledo JP, Díaz-San Andrés B, et al. Inflammatory pseudotumour of the spleen associated with splenic tuberculosis. World J Gastrointest Surg 6 (2014): 248-252.

10. Kawaguchi T, Mochizuki K, Kizu T, et al. Inflammatory pseudotumor of the liver and spleen diagnosed by percutaneous needle biopsy. World J Gastroenterol 18 (2012): 90-95.

11. Okura N, Mori K, Morishita Y, et al. Inflammatory pseudotumor of the intrapancreatic accessory spleen: computed tomography and magnetic resonance imaging findings. Jpn J Radiol 30 (2012): 171-175.

This article is an open access article distributed under the terms and conditions of the

$\underline{\text { Creative Commons Attribution (CC-BY) license 4.0 }}$ 